\title{
Modeling of Tool Wear in Vibration Assisted Nano Impact-Machining by Loose Abrasives
}

\author{
Sagil James and Murali M. Sundaram \\ Department of Mechanical and Materials Engineering, University of Cincinnati, Cincinnati, OH 45221, USA \\ Correspondence should be addressed to Murali M. Sundaram; murali.sundaram@uc.edu
}

Received 17 May 2014; Accepted 9 September 2014; Published 20 October 2014

Academic Editor: Tuğrul Özel

Copyright ( 2014 S. James and M. M. Sundaram. This is an open access article distributed under the Creative Commons Attribution License, which permits unrestricted use, distribution, and reproduction in any medium, provided the original work is properly cited.

\begin{abstract}
Vibration assisted nano impact-machining by loose abrasives (VANILA) is a novel nanomachining process that combines the principles of vibration assisted abrasive machining and tip-based nanomachining, to perform target specific nanoabrasive machining of hard and brittle materials. An atomic force microscope (AFM) is used as a platform in this process wherein nanoabrasives, injected in slurry between the workpiece and the vibrating AFM probe which is the tool, impact the workpiece and cause nanoscale material removal. The VANILA process are conducted such that the tool tip does not directly contact the workpiece. The level of precision and quality of the machined features in a nanomachining process is contingent on the tool wear which is inevitable. Initial experimental studies have demonstrated reduced tool wear in the VANILA process as compared to indentation process in which the tool directly contacts the workpiece surface. In this study, the tool wear rate during the VANILA process is analytically modeled considering impacts of abrasive grains on the tool tip surface. Experiments are conducted using several tools in order to validate the predictions of the theoretical model. It is seen that the model is capable of accurately predicting the tool wear rate within $10 \%$ deviation.
\end{abstract}

\section{Introduction}

Well-known tip-based nanomachining processes such as nanoscratching and nanoindentation involve direct tool contact with the workpiece and result in high tool wear. Vibration assisted nano impact-machining by loose abrasives (VANILA) process, a noncontact vibration tip-assisted nanomachining process that uses loose abrasives, has been investigated and can be used to perform target specific impact-based machining of nanoscale features on hard and brittle materials, such as glass and ceramics materials $[1$, 2]. This process uses an AFM platform in which a slurry of nanodiamond abrasive particles is introduced between the tool and the workpiece. The machining is conducted in tapping mode where the tool vibrates at resonance within the slurry of abrasive nanoparticles. The impact of the tool along with the acoustic field generated moves the abrasive grain towards the workpiece surface causing a high velocity impact and material removal. A schematic of the VANILA process is shown in Figure 1.
The feasibility of nanoscale machining using the VANILA process was successfully demonstrated on hard and brittle materials such as borosilicate glass and silicon. Patterns of nanocavities were successfully machined to demonstrate the controllability and repeatability of the process as shown in Figure 2 [2].

The VANILA process uses an AFM probe as the vibrating tool to impact the nanoabrasive grains which causes material removal at nanoscale. Earlier studies using scanning electron microscopy (SEM) imaging have shown that tool wear in the VANILA process is comparatively lower than that in other nanomachining processes such as nanoscratching and nanoindentation involving direct tool contact with the workpiece (Figure 3) [2]. The accuracy and precision of any tip-based nanomachining process is limited by the tip's sharpness, material, and geometry [3]. The level of precision of the nanomachined features continuously degrades during the machining process as the probe tip inevitably wears resulting in an increase of contact diameter and forces acting on it [3]. Establishing the point at which the tool is considered 


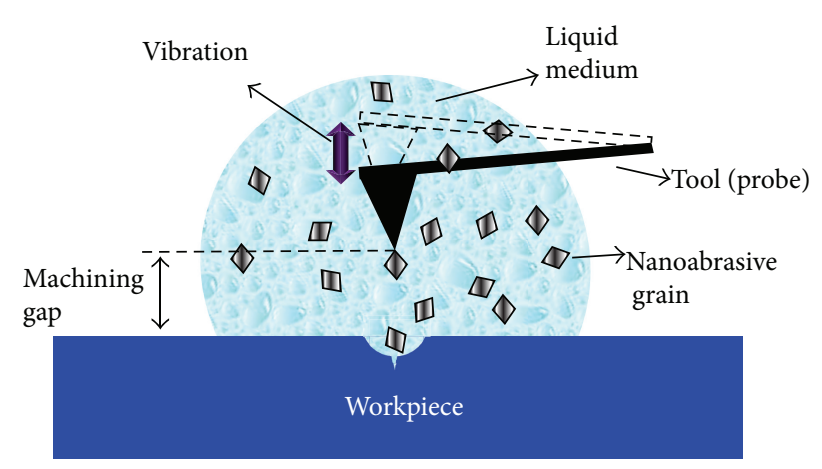

FIgURE 1: Schematic diagram of the VANILA process.

worn is important, since, after this point, machining results are no longer acceptable [4]. Thus quantification of tool wear to predict the tool life is of great significance. This study aims to develop a theoretical model to predict the tool wear in the VANILA process.

\section{Mechanism of Tool Wear during VANILA Process}

The nanoscale wear of AFM tip often refers to the volumetric wear of the tip due to the height loss and varying tip radius. The AFM probes, in general, have a sharp pyramidal tip of the order of few nanometers. The wear during operation causes tip to become blunt with larger tip. Studies have found that the tip shape and geometry significantly influence its deformation behaviors at nanoscales [5-7]. A review of the available literature reveals that that AFM tip wear occurs through multiple wear mechanisms $[8,9]$ such as abrasive and adhesive wear [10], low-cycle fatigue and material fracture [10], plastic deformation [11], coating failure, corrosive wear, and tribochemical wear [11], and thermochemical wear [12]. Recently, abrasive wear of AFM tips has been studied at atomic scales and it is found that the damaged surface is smoothly modified [13]. This basic abrasive-wear mechanism is often termed as gradual wear due to atom-by-atom attrition [14], whereby single atoms or cluster makes up the wear debris.

During the VANILA process, the resonating tool tip repeatedly impacts the nanoabrasive grains suspended in the slurry. The tool tip wear caused by these nanoscale impacts in rapid succession would lead to considerable size effects both in the length and in the time scales. Decohesion forces or pull-out forces become significant which may lead to gradual removal of atoms from the tool tip. This phenomenon is further supported by the fact that, at such lesser tool dimensions, the energy required to break the atomic bonds decreases due to bond stretching [14]. Based on the literature study, it is understood that, although several mechanisms could be involved, wear due to gradual atom-by-atom loss would be the most relevant wear mechanism causing the tool wear in the VANILA process [14]. Thus, based on this information, a wear model is developed to predict and quantify the tool wear during the VANILA process. Other wear mechanisms that could affect the tool wear process are ductile mode plastic deformation and nanoscale cracking [15]. However, these mechanisms would be more relevant when the tool tip size is considerably larger than the abrasive grain size.

\section{Modeling of Tool Wear in VANILA Process}

During the VANILA process, the tool tip and workpiece surface are maintained at a distance. The tool wears due to the impact of nanoabrasive grains. For the purpose of analytical modeling of tool wear, the following simplification assumptions are made.

3.1. Simplification Assumptions. The simplification assumptions made in developing the theoretical model are listed as follows.

(1) The abrasive grains are assumed to be spherical and rigid and remain intact throughout the machining process.

(2) Abrasive nanoparticle and tool material are electrically and chemically neutral and the electrostatic effects during impact are neglected.

(3) The effects of surface adhesion and interactions outside the region of contact are not considered in this study.

(4) For the theoretical modeling, the tool tip is assumed to be flat. The change in profile of the tool tip during the machining process is not considered in this study.

(5) The effects of abrasive grain impact on the sides of the tool tip are not considered in this study.

(6) The collisions between the abrasives grains are assumed to be perfectly elastic.

3.2. Impact Velocity and Forces. During the VANILA process, the abrasive grains moving in the slurry medium impact the tool which is excited at resonance. The resultant velocity of impact $\left(\mathbf{V}_{\mathbf{I}}\right)$ has two components: (a) velocity of vibrating tool $\left(\mathbf{V}_{\mathbf{t}}\right)$ and (b) velocity of abrasive grain $\left(\mathbf{V}_{\mathbf{a}}\right)$. The motion of tool tip can be expressed as $A_{t} e^{i(\omega t+\pi / 2)}$. The phase associated with the total force can be considered as $\pi / 2$ due to the fact that machining is conducted at resonance frequency and since on resonance the oscillations of the cantilever follow the total force with a phase delay of $\pi / 2$ [16]. Average velocity of the tool tip $\left(\mathbf{V}_{\mathbf{t}}\right)$ can be expressed as

$$
\mathbf{V}_{\mathbf{t}}=0.5 \omega A_{t}=\pi f_{t} A_{t}
$$

The velocity of the nanoabrasive grain within the fluid medium can be considered to be equal to the velocity of the fluid molecule. The acoustic streaming motion generated by the acoustic field near the tool tip results in the motion of the fluid slurry between the tool tip and the workpiece surface. The nanoparticle within the slurry is transported along with the streaming fluid molecules and causes impact with the 


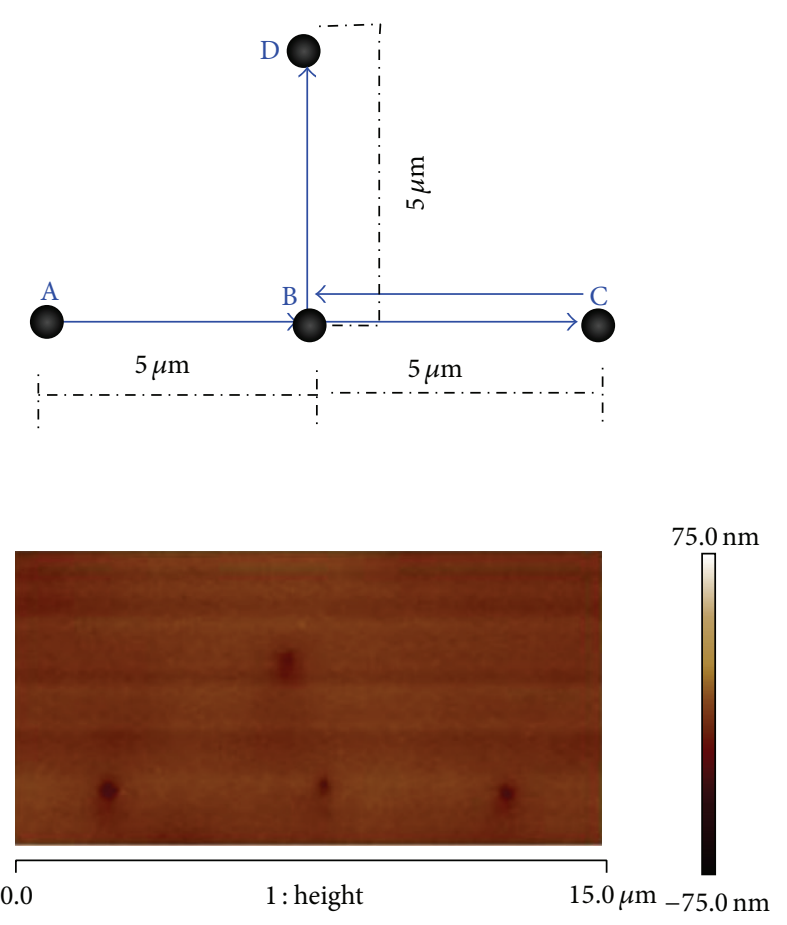

(a)
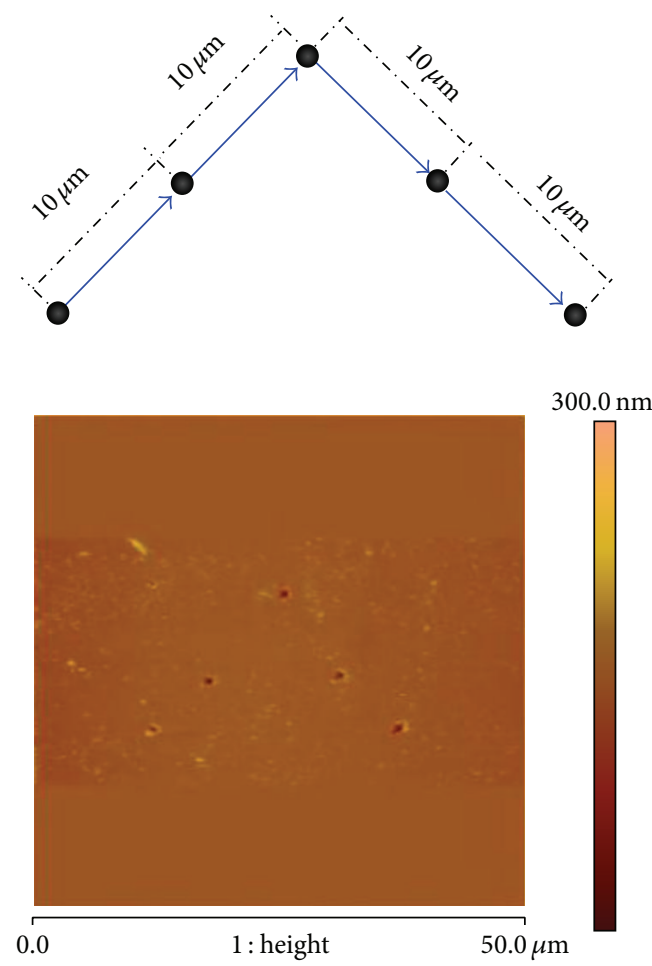

(b)

FIGURE 2: Pattern design and AFM image of nanocavity pattern machined using the VANILA process (a) on silicon substrate [2] and (b) on borosilicate glass substrate.

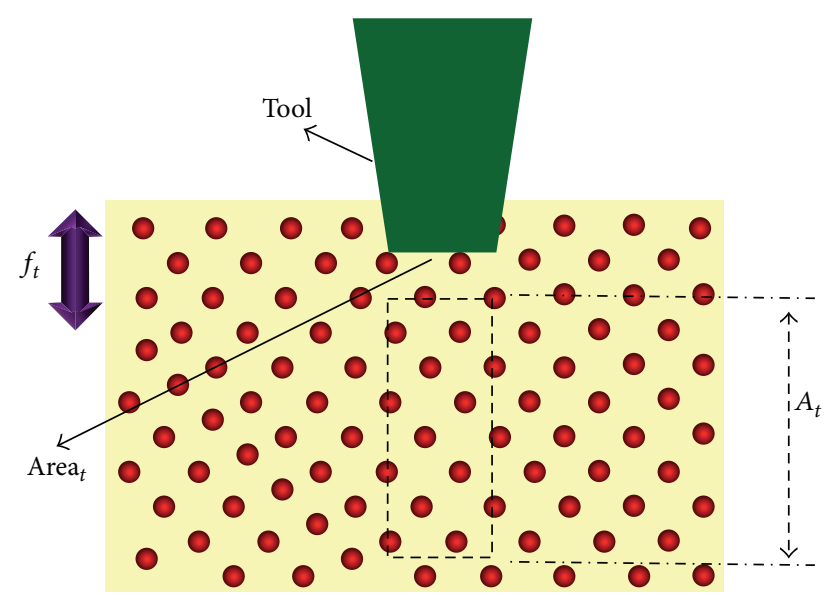

FIGURE 3: Schematic showing machining zone in VANILA process.

tool tip surface. The nanoabrasive grain velocity $\left(\mathbf{V}_{\mathbf{a}}\right)$ can be estimated using the relation [17]

$$
\mathbf{V}_{\mathbf{a}}=\left[\frac{I_{t}}{\rho_{f l} c_{f l}}\right]^{1 / 2},
$$

where $I_{t}$ is the vibration intensity of the acoustic waves generated at the tool tip and can be calculated by dividing the acoustic power by the area of the radiating surface which is the tool tip surface area [18]:

$$
I_{t}=\frac{P_{t}}{\left(\pi R_{t}^{2}\right)} .
$$

The acoustic power generated at the tip which is excited at its resonant frequency can be found as [19]

$$
P_{t}=\frac{\pi k_{t} A_{t}^{2} f_{t}}{Q_{t}} .
$$

The impact velocity $\mathbf{V}_{\mathbf{I}}$ can be expressed as

$$
\mathrm{V}_{\mathrm{I}}=\mathrm{V}_{\mathrm{t}}+\mathrm{V}_{\mathrm{a}} \text {. }
$$

According to the law of conservation of momentum, the impact force $\left(F_{I}\right)$ on the tool tip surface due to the impact with the abrasive nanoparticles can be expressed as [20]

$$
\mathbf{F}_{\mathbf{I}}=\frac{m_{a} \mathbf{V}_{\mathbf{I}} \sin \theta}{\Delta t}
$$

where $\theta$ is the angle of impact and $\Delta t$ is the response time defined as

$$
\Delta t=\frac{2 \rho_{f l} R_{a}^{2}}{9 \rho_{a} \nu_{f l}} .
$$

The response time can be considered as the time scale over which the particles respond to changes in flow conditions [20]. Also, the mass term in the expression for $F_{I}$ only 
considers the abrasive grain mass since the tool tip mass is much larger than the grain mass.

3.3. Establishing Threshold Criteria. When the impact force $F_{I}$ is larger than the critical pull-out force $F_{c}$, the tool atoms are able to pass through a critical energy barrier resulting in gradual atom-by-atom attrition [14]. The critical pull-out force can be expressed as [21]

$$
\mathbf{F}_{\mathbf{c}}=\frac{3}{2} \pi w R^{*}
$$

where $w$ is the Dupré energy of adhesion and $R^{*}$ is effective tool tip radius which is given by [21]

$$
R^{*}=\left(\frac{1}{R_{a}}+\frac{1}{R_{t}}\right)^{-1} \text {. }
$$

The effective radius term would enable modeling the tool wear in the VANILA process at various instants of wear. This formulation of $R^{*}$ is commonly used in classical Hertzian contact theory to determine the contact radius between the impacting bodies.

3.4. Estimation of Tool Wear Rate. Tool wear rate TWR is proportional to the product of volume of tool removed $\tau_{t}$ by single impact, the total number of active abrasive grains $n_{a}$, and the frequency of vibration $f_{t}$ :

$$
\mathrm{TWR} \propto \tau_{t} * n_{a} * f_{t} .
$$

Introducing a proportionality factor $\lambda$ termed as wear coefficient, the tool wear rate can be determined as

$$
\mathrm{TWR}=\lambda * \tau_{t} * n_{a} * f_{t}
$$

3.5. Volume Removed by Single Impact. Since the wear happens at atomistic scales, the classical theories of mechanics may not be applicable. A quantum mechanism based approach is thus used to estimate the volume $\tau_{t}$ removed by single impact of the abrasive grain. The tool volume removed $\tau_{t}$ due to interaction with single nanoabrasive grain can be deduced by the D'Acunto model [13, 22, 23]:

$$
\tau_{t}=\Delta t * n_{\text {atom }} * w_{\text {atom }} * \operatorname{vol}_{\text {atom }},
$$

where $n_{\text {atom }}$ is the number of atoms passing through the energy barrier, $w_{\text {atom }}$ is the transition rate, and $\operatorname{vol}_{\text {atom }}$ is the individual atomic volume. $\Delta t$ corresponds to the time of interaction between the abrasive grain and the tool which is described in earlier section of this paper. The term $\left(n_{\text {atom }} *\right.$ $\left.w_{\text {atom }}\right)$ can be calculated using the well-known Arrhenius kinetics [24] as follows [22]:

$$
w_{\text {atom }} * n_{\text {atom }}=f_{\text {atom }} * \overline{\rho_{t}} * A_{c} * e^{-\Delta E / k_{B} T},
$$

where $\Delta E$ is the effective activation energy barrier, $k_{B}$ is the Boltzmann constant, $\overline{\rho_{t}}$ is the surface atomic density of the tool tip, $A_{c}$ is the area of contact between the tool and abrasive grain which is circular region having diameter $R^{*}$; that is,
$A_{c}=\pi R^{* 2}$. The term $f_{\text {atom }}$ corresponds to the attempt frequency or oscillation frequency.

The activation energy barrier for the atoms to be removed from the tool is considerably reduced by the bond stretching due to impact of the abrasive grain. Thus the effective activation energy barrier $\Delta E$ is the difference of the activation energy $E_{\text {act }}$ of the tool material and the energy of impact $\mathbf{E}_{\mathbf{i}}=(1 / 2) m_{a} \mathbf{V}_{\mathbf{I}}^{2}$ of the abrasive grain $\left(\Delta E=\mathbf{E}_{\text {act }}-\mathbf{E}_{\mathbf{i}}\right)$.

3.6. Number of Active Abrasives. $n_{a}$ is the number of active abrasive grains in the machining zone which can be derived as follows. The vibrating tool impacts the abrasive grains within the volume of an imaginary cylinder having diameter equal to tool tip diameter and height equal to the amplitude of vibration of the tool as shown in dotted line in Figure 3. It is to be noted that this figure is not drawn to the actual scale and its purpose is to show the method of determining the active abrasive grains.

The volume of active abrasive particles within this imaginary cylinder can be expressed as

$$
\text { Volume }=\operatorname{Area}_{t} A_{t} c_{z}
$$

where Area $_{t}$ is the cross-sectional area of tool tip given by Area $_{t}=\pi R_{t}^{2}, c_{z}$ is the slurry volume concentration, and $A_{t}$ is the amplitude of tool vibration.

The volume of active abrasive particles within the imaginary cylinder can also be expressed as the product of the number of abrasive nanoparticles $\left(n_{a}\right)$ within the imaginary cylinder and volume of a single grain:

$$
\text { Volume }=n_{a} * \frac{4 \pi R_{a}^{3}}{3}
$$

Using the above equalities, $n_{a}$ can be calculated as

$$
n_{a}=\frac{\left(3 R_{t}^{2} A_{t} c_{z}\right)}{\left(4 R_{a}^{3}\right)} .
$$

3.7. Tool Wear Rate Model. Substituting the expressions for $n_{a}$ and $\tau_{t}$ in (11), the tool wear rate TWR can be expressed as

$$
\begin{aligned}
\operatorname{TWR}= & \frac{\pi \lambda}{6} * f_{t} A_{t} * \frac{\left(R_{t} R^{*}\right)^{2}}{R_{a}} c_{z} * \frac{\rho_{f l}}{\rho_{a} \nu_{f l}} * \overline{\rho_{t}} \\
& * f_{\text {atom }} * \operatorname{vol}_{\text {atom }} * e^{-\Delta E / k_{B} T} .
\end{aligned}
$$

\section{Experimental Verification}

The VANILA process experiments are performed using several commercially available tapping mode AFM probes and $10 \mathrm{~nm}$ sized nanodiamond abrasive grains suspended in deionized water-based slurry. The tools used are made of commercially available tapping mode probes (NSC16 series MikroMasch make) made of $n$-type silicon having a tip diameter in the range of $8-10 \mathrm{~nm}$ and is excited at its resonance frequency $(5-10 \mathrm{KHz}$ range). The tool tip is vibrated at its resonance frequency using the cantilever tuning operation 


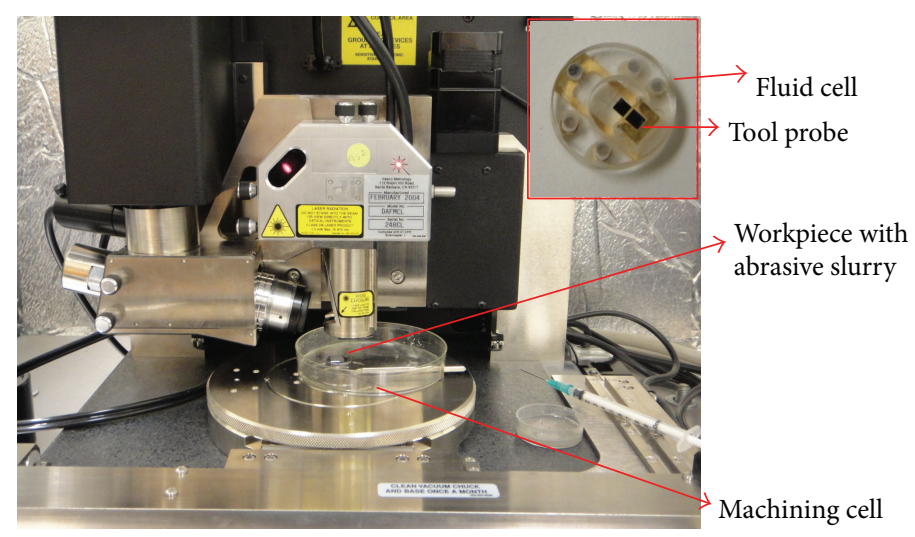

(a)

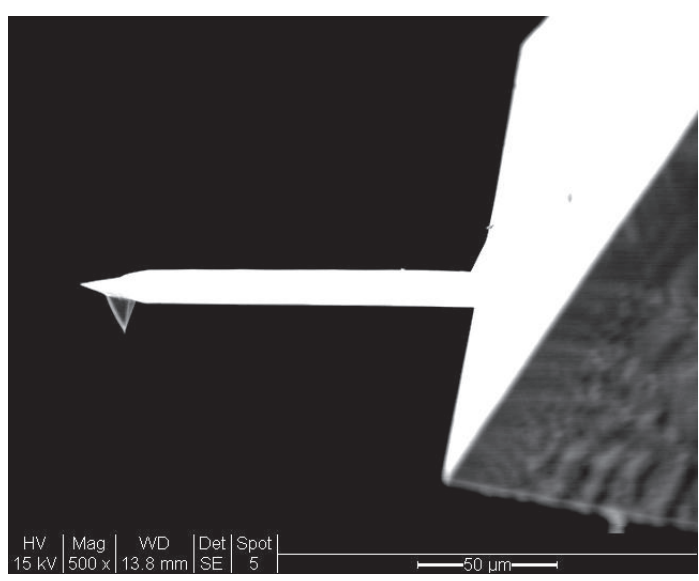

(c)

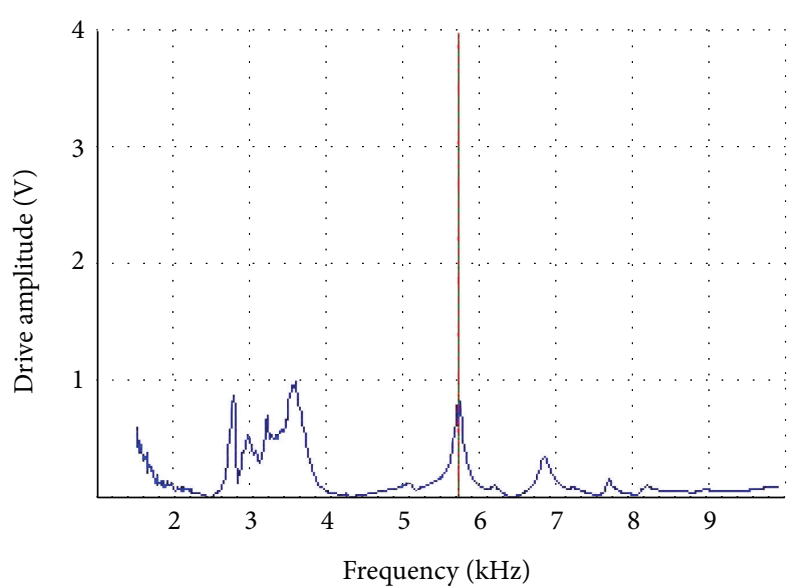

(b)

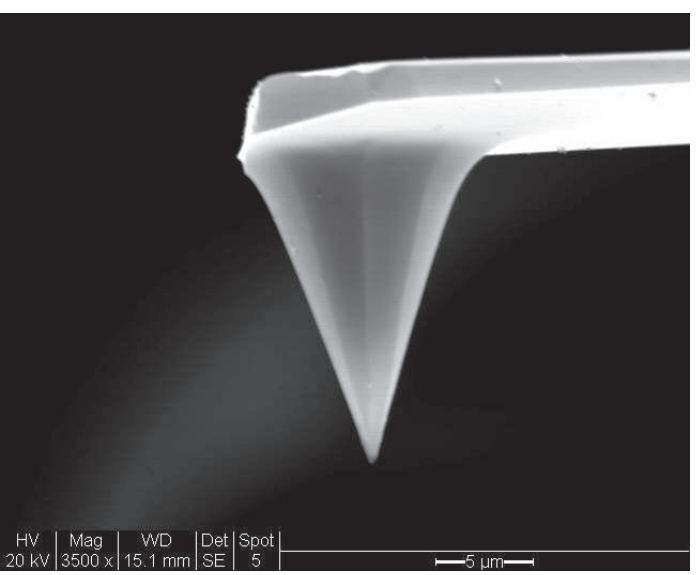

(d)

Figure 4: (a) Experimental setup (Inset: fluid cell), (b) cantilever tuning curve, (c) tool probe used for machining, and (d) zoomed view of tool tip.

in the Nanoscope IIIa software of the AFM. Representative drive amplitude versus frequency curve during the cantilever tuning for VANILA process is shown in Figure 4(b). The resonance frequency is chosen from the peaks shown during the cantilever tuning.

The maximum vibration amplitude of the tool $\left(A_{t}\right)$ is approximately $200 \mathrm{~nm}$ with a quality factor $\left(Q_{t}\right)$ ranging from 1to 50 and spring constant $\left(k_{t}\right) 0.5-45 \mathrm{~N} / \mathrm{m}$. Properties of deionized water that are considered in this study are dynamic viscosity $\left(v_{f l}\right)=0.00078 \mathrm{Kg} / \mathrm{m} \cdot \mathrm{s}$, density $\left(\rho_{f l}\right)=$ $1000 \mathrm{Kg} / \mathrm{m}^{3}$, and speed of sound in water $(c)$ as $1500 \mathrm{~m} / \mathrm{s}$. AFM (Veeco Dimension 3100 with Nanoscope III) is used to perform experiments along with a direct drive fluid cantilever holder (DDFCH) for holding the tool. The tool tip is vibrated at its resonant frequency. Polished silicon wafers are used as the workpiece substrate and the abrasive slurry is introduced on the workpiece surface using a syringe. The tool tip is maintained at a distance of 100-200 $\mathrm{nm}$ above the substrate surface to avoid contact, while the amplitude of vibration is approximately $100 \mathrm{~nm}$. The machining set-up used for the VANILA process is shown in Figure 4(a). Figures 4(c) and 4(d) show the tool tip (and enlarged view) used for the
TABLE 1: Experimental conditions for VANILA process.

\begin{tabular}{lc}
\hline Machine & AFM dimension 3100 \\
Abrasive & $10 \mathrm{~nm}$ sized diamond nanoparticles \\
Tool & Silicon with $\sim 8 \mathrm{~nm}$ tip radius \\
Liquid medium & Deionized water \\
Slurry concentration & 0.5 vol. conc $\%$ \\
Temperature & $300 \mathrm{~K}$ \\
\hline
\end{tabular}

VANILA process. The common experimental conditions are listed in Table 1.

Each tip is used to machine several nanocavities before it is analyzed using scanning electron microscope (SEM) images (make - FEI XL30 ESEM). In order to determine the wear volume with nanoscale precision, SEM images are analyzed and relevant tool dimensions such as its tip length and radius are estimated as shown in Figure 5. Using this information, three-dimensional computer models of the tool tips are created using 3D modeling software SolidWorks in Figure 5(c). The model is sliced at a specified length which is obtained from the SEM images of the worn tip as 


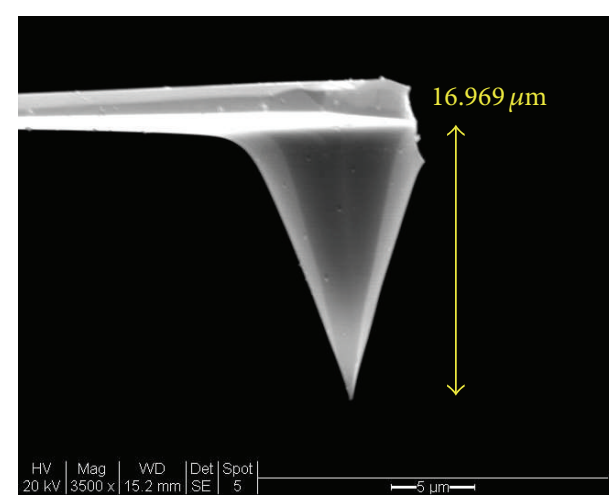

(a) Unused tool tip

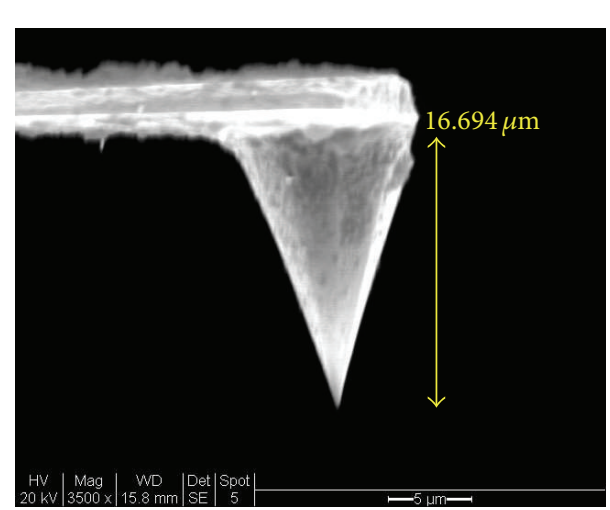

(b) Worn tool tip after $300 \mathrm{~s}$

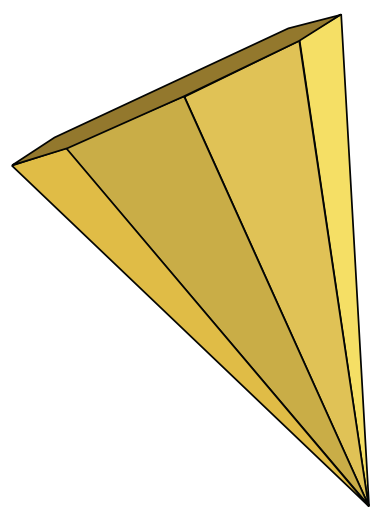

(c) CAD model of tool tip

FIGURE 5: SEM images and solid model of tool tip used for VANILA process.

shown in Figure 5(b). The volume of the slice of the tool is geometrically determined using the mass properties tool in SolidWorks. Experimental tool wear rate (TWR) is estimated by dividing the calculated wear volume by the total time spent by the resonating tip in the abrasive slurry. During experimentation, the time is measured using a stopwatch.

In order to calculate the tool wear rate TWR, the wear coefficient $\lambda$ needs to be determined empirically. For this purpose, a series of experiments are conducted and the experimental wear rates are calculated. Individual wear coefficient values are then determined by dividing the experimental wear rate values by the theoretical predictions (assuming $\lambda$ as unity). An average of the individual $\lambda$ values (9601, 11034, and 8034) is then considered as the final $\lambda$ value for the tool wear rate model. During this process, the value of the wear coefficient $\lambda$ is estimated to be approximately 9500 .

In these calculations, the surface atomic density of the tool tip $\overline{\rho_{t}}$ is taken as $10^{16}$ atoms $/ \mathrm{cm}^{2}$ [25], the response time is approximately $10^{-12}$ seconds, and the attempt frequency $f_{\text {atom }}$ has typical values of $10^{13} \mathrm{cps}$ for silicon [26]. The atomic volume of silicon ( $\mathrm{vol}_{\text {atom }}$ ) is taken as $0.02 \mathrm{~nm}^{3}$ with an activation energy $E_{\text {act }}$ value of $0.9 \mathrm{eV}$ [14]. Based on the experimental conditions, the impact velocity is approximately in the range of $2-3 \mathrm{~m} / \mathrm{s}$ and the impact force is estimated in the range of $20-50 \mathrm{nN}$ assuming the angle of impact to be normal. The Dupré energy of adhesion for silicon is taken as $4 \mathrm{~mJ} / \mathrm{m}^{2}$ [27] and the critical pull-out force $F_{c}$ is estimated to be $0.19 \mathrm{nN}$ which is lower than the force of impact and thus the wear could progress. The duration of machining for the tools is varied between $100 \mathrm{~s}$ and $1200 \mathrm{~s}$ where the tool is used to machine multiple nanoscale cavities on the workpiece. Figure 6 shows the plot between the theoretically predicted TWR and the experimental TWR for machining durations ranging from $100 \mathrm{~s}$ to $1200 \mathrm{~s}$ for a $\lambda$ value of 9500 .

From the study, it is seen that during the VANILA process, the typical tool wear rates are in the order of $10^{3} \mathrm{~nm}^{3} / \mathrm{s}$ compared to the material removal rates (MRR) from the workpiece which is in the order of $10^{5} \mathrm{~nm}^{3} / \mathrm{s}$ [28]. From the plot, it is seen that the TWR model is capable of predicting the tool wear rates within $10 \%$ accuracy. It is seen that the

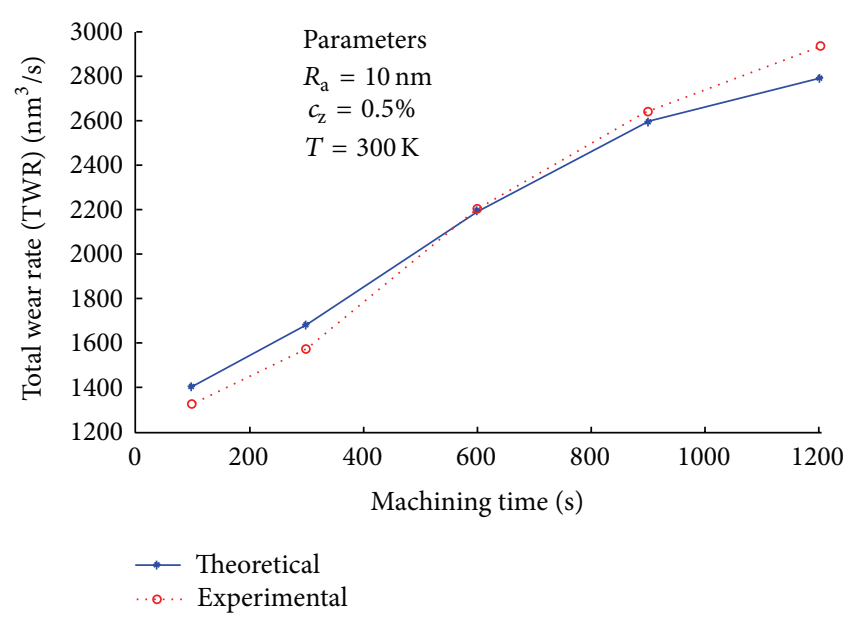

FIgURE 6: Plot showing tool wear rates from experiments versus theoretical predictions for varying machining duration.

tool wear increases gradually with the machining duration. This could be due to the fact that, as the machining duration increases, the number of active abrasive grains which affect the tool wear process increases resulting in increased tool wear rates. For the theoretical calculation of TWR, final tool tip radius value (from the SEM images of worn tool tips) is used to calculate the $R^{*}$ value.

\section{Conclusion}

In this study, the tool wear involved in the VANILA process is studied using analytical modeling and experimental methods. Tool wear rates are estimated to be in the order of $10^{3} \mathrm{~nm}^{3} / \mathrm{s}$ during the machining process for tool tips made of silicon. In order to validate the model, experiments are performed with different tools for varying machining durations. Reasonably good quantitative correlations (within $10 \%$ ) are obtained between the model predictions and the corresponding experimental observations. 


\section{Nomenclature}

\section{Tool Variables}

$R_{t}: \quad$ Radius of tool tip (nm)

Area ${ }_{t}$ : Cross-sectional area of tool tip $\left(\mathrm{nm}^{2}\right)$

$f_{t}$ : Frequency of vibration of tool $(\mathrm{KHz})$

$A_{t}: \quad$ Amplitude of vibration of tool (nm)

$\mathbf{V}_{\mathbf{t}}$ : Velocity of tool tip $(\mathrm{m} / \mathrm{s})$

$Q_{t}: \quad$ Quality factor of tool

$k_{t}$ : $\quad$ Spring constant of tool

$P_{t}: \quad$ Power generated at tool tip

$I_{t}$ : Acoustic vibration intensity.

\section{Fluid Variables}

$\rho_{f l}$ : Density of fluid $\left(\mathrm{kg} / \mathrm{m}^{3}\right)$

$v_{f l}$ : Kinematic viscosity of fluid $\left(\mathrm{m}^{2} / \mathrm{s}\right)$

$c_{f l}$ : Acoustic velocity in liquid medium $\left(\mathrm{kg} / \mathrm{m}^{3}\right)$.

\section{Abrasive Grain Variables}

$R_{a}:$ Radius of abrasive grain $(\mathrm{nm})$

$R^{*}$ : Effective radius ( $\mathrm{nm}$ )

$\rho_{a}$ : Density of abrasive grain $\left(\mathrm{kg} / \mathrm{m}^{3}\right)$

$m_{a}$ : Mass of abrasive grain $(\mathrm{kg})$

$c_{z}$ : Volume concentration of abrasive grain slurry (\%)

$\mathbf{V}_{\mathbf{t}}$ : Impact velocity of abrasive grain $(\mathrm{m} / \mathrm{s})$.

\section{Process Parameters}

$w$ : Dupré energy of adhesion $\left(\mathrm{J} / \mathrm{m}^{2}\right)$

$\Delta t: \quad$ Response time (s)

$\mathbf{V}_{\mathbf{t}}: \quad$ Impact velocity $(\mathrm{m} / \mathrm{s})$

$\mathbf{F}_{\mathbf{t}}$ : Impact force $(\mathrm{nN})$

$\mathbf{F}_{\mathrm{c}}: \quad$ Critical pull-out force $(\mathrm{nN})$

$\lambda$ : Wear coefficient

$\Delta E: \quad$ Effective activation energy barrier $(\mathrm{J})$

$E_{\text {act }}: \quad$ Activation energy $(\mathrm{J})$

$E_{i}: \quad$ Kinetic energy of impact (J)

$\overline{\rho_{t}}: \quad$ Surface atomic density of the tool tip

$A_{c}$ : $\quad$ Area of contact

$f_{\text {atom }}$ Attempt frequency or oscillation frequency

vol $_{\text {atom }}$ : Volume of tool atom

$\tau_{t}: \quad$ Volume removed in a single impact $\left(\mathrm{nm}^{3}\right)$

$\theta: \quad$ Angle of impact (o)

$k_{B}: \quad$ Boltzmann constant $(\mathrm{J} / \mathrm{K})$

$T: \quad$ Temperature $(\mathrm{K})$

$n_{\text {atom: }}$ Number of atoms passing the energy barrier

$w_{\text {atom }}$ : Transition rate

$n_{a}: \quad$ Number of active abrasive grains.

\section{Conflict of Interests}

The authors declare that there is no conflict of interests regarding the publication of this paper.

\section{Acknowledgments}

This material is based upon work supported by the National Science Foundation under Grant nos. CMMI-1137968, CMMI-1120382, and CBET-1239779. The research facilities provided by the Advanced Material Characterization Center at the University of Cincinnati are acknowledged.

\section{References}

[1] M. M. Sundaram and S. James, "Vibration assisted nano abrasive machining," in Proceedings of the International Conference on Precision, Meso, Micro, and Nano Engineering, College of Engineering, Pune, India, 2011.

[2] S. James and M. M. Sundaram, "A feasibility study of vibrationassisted nano-impact machining by loose abrasives using atomic force microscope," Journal of Manufacturing Science and Engineering, vol. 134, no. 6, Article ID 61014, 2012.

[3] R. Agrawal, N. Moldovan, and H. D. Espinosa, "An energybased model to predict wear in nanocrystalline diamond atomic force microscopy tips," Journal of Applied Physics, vol. 106, no. 6, Article ID 064311, 2009.

[4] G. M. Robinson, M. J. Jackson, and M. D. Whitfield, "A review of machining theory and tool wear with a view to developing micro and nano machining processes," Journal of Materials Science, vol. 42, no. 6, pp. 2002-2015, 2007.

[5] C. Lu, Y. Gao, G. Michal, N. N. Huynh, H. T. Zhu, and A. K. Tieu, "Atomistic simulation of nanoindentation of iron with different indenter shapes," Proceedings of the Institution of Mechanical Engineers Part J: Journal of Engineering Tribology, vol. 223, no. 7, pp. 977-984, 2009.

[6] K. Komvopoulos and W. Yan, "Molecular dynamics simulation of single and repeated indentation," Journal of Applied Physics, vol. 82, no. 10, pp. 4823-4830, 1997.

[7] P.-Z. Zhu, Y.-Z. Hu, H. Wang, and T.-B. Ma, "Study of effect of indenter shape in nanometric scratching process using molecular dynamics," Materials Science and Engineering A, vol. 528, no. 13-14, pp. 4522-4527, 2011.

[8] H.-J. Kim, S.-S. Yoo, and D.-E. Kim, "Nano-scale wear: a review," International Journal of Precision Engineering and Manufacturing, vol. 13, no. 9, pp. 1709-1718, 2012.

[9] J. Liu, J. K. Notbohm, R. W. Carpick, and K. T. Turner, "Method for characterizing nanoscale wear of atomic force microscope tips," ACS Nano, vol. 4, no. 7, pp. 3763-3772, 2010.

[10] A. Khurshudov and K. Kato, "Wear of the atomic force microscope tip under light load, studied by atomic force microscopy," Ultramicroscopy, vol. 60, no. 1, pp. 11-16, 1995.

[11] M. L. Bloo, H. Haitjema, and W. O. Pril, "Deformation and wear of pyramidal, silicon-nitride AFM tips scanning micrometresize features in contact mode," Measurement: Journal of the International Measurement Confederation, vol. 25, no. 3, pp. 203-211, 1999.

[12] K. Cheng, X. Luo, R. Ward, and R. Holt, "Modeling and simulation of the tool wear in nanometric cutting," Wear, vol. 255, no. 7-12, pp. 1427-1432, 2003.

[13] M. D'Acunto, “Theoretical approach for the quantification of wear mechanisms on the nanoscale," Nanotechnology, vol. 15, no. 7, pp. 795-801, 2004.

[14] B. Gotsmann and M. A. Lantz, "Atomistic wear in a single asperity sliding contact," Physical Review Letters, vol. 101, no. 12, Article ID 125501, 2008. 
[15] S. James and M. M. Sundaram, "A molecular dynamics study of the effect of impact velocity, particle size and angle of impact of abrasive grain in the Vibration Assisted Nano Impactmachining by Loose Abrasives," Wear, vol. 303, no. 1-2, pp. 510518, 2013.

[16] O. Sahin, C. F. Quate, O. Solgaard, and A. Atalar, "Resonant harmonic response in tapping-mode atomic force microscopy," Physical Review B: Condensed Matter and Materials Physics, vol. 69, no. 16, Article ID 165416, 2004.

[17] Q. Qi and G. J. Brereton, "Mechanisms of removal of micronsized particles by high-frequency ultrasonic waves," IEEE Transactions on Ultrasonics, Ferroelectrics, and Frequency Control, vol. 42, no. 4, pp. 619-629, 1995.

[18] D. M. VandenBurg, The Sonochemical Remediation of Phthalate Esters: An Investigation into Products and Kinetics, University of Bath, Bath, UK, 2011.

[19] J. P. Cleveland, B. Anczykowski, A. E. Schmid, and V. B. Elings, "Energy dissipation in tapping-mode atomic force microscopy," Applied Physics Letters, vol. 72, no. 20, pp. 2613-2615, 1998.

[20] S. M. Booij, Fluid Jet Polishing: Possibilities and Limitations of a New Fabrication Technique, 2003.

[21] D. Maugis, "Adhesion of spheres: the JKR-DMT transition using a Dugdale model," Journal of Colloid And Interface Science, vol. 150, no. 1, pp. 243-269, 1992.

[22] R. Bassani and M. D’Acunto, "Nanotribology: tip-sample wear under adhesive contact," Tribology International, vol. 33, no. 7, pp. 443-452, 2000.

[23] T. D. B. Jacobs, B. Gotsmann, M. A. Lantz, and R. W. Carpick, "On the application of transition state theory to atomic-scale wear," Tribology Letters, vol. 39, no. 3, pp. 257-271, 2010.

[24] K. J. Laidler, "The development of the arrhenius equation," Journal of Chemical Education, vol. 61, no. 6, pp. 494-498, 1984.

[25] M. D’Acunto, “Controlling wear on nanoscale," in Scanning Probe Microscopy in Nanoscience and Nanotechnology, pp. 647686, Springer, 2010.

[26] C. M. Bertoni, V. Bortolani, C. Calandra, and E. Tosatti, "Dielectric matrix and phonon frequencies in silicon," Physical Review Letters, vol. 28, no. 24, pp. 1578-1581, 1972.

[27] V. V. Tsukruk and V. N. Bliznyuk, "Adhesive and friction forces between chemically modified silicon and silicon nitride surfaces," Langmuir, vol. 14, no. 2, pp. 446-455, 1998.

[28] S. James and M. Sundaram, "Modeling of material removal rate in vibration assisted nano impact-machining by loose abrasives," Journal of Manufacturing Science and Engineering, 2014. 

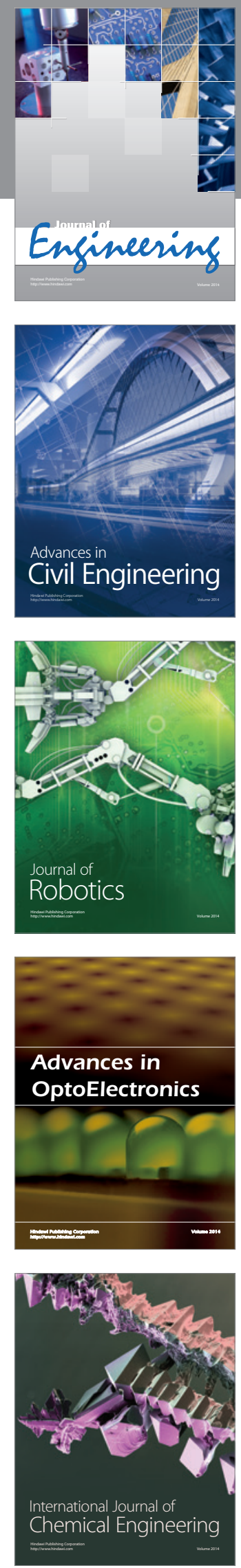

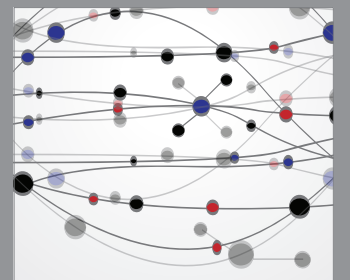

The Scientific World Journal
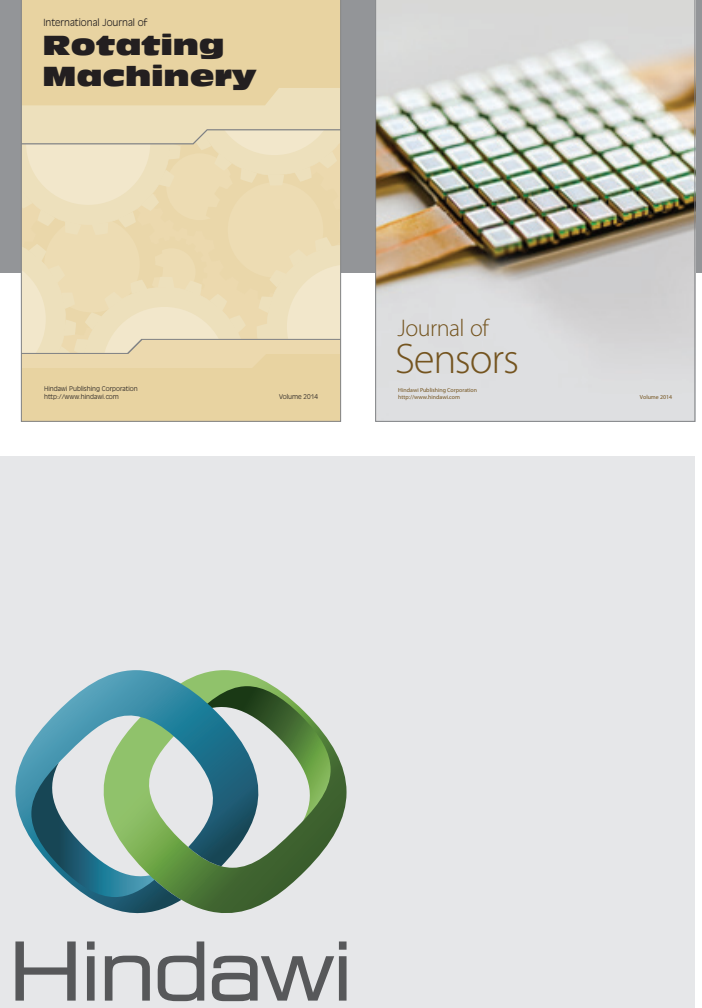

Submit your manuscripts at http://www.hindawi.com
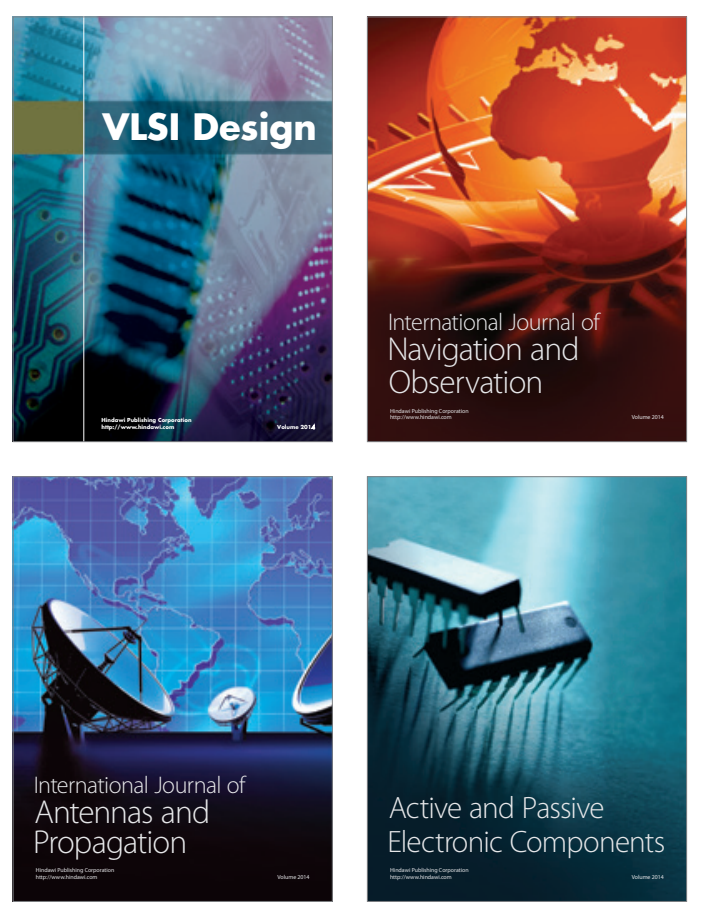
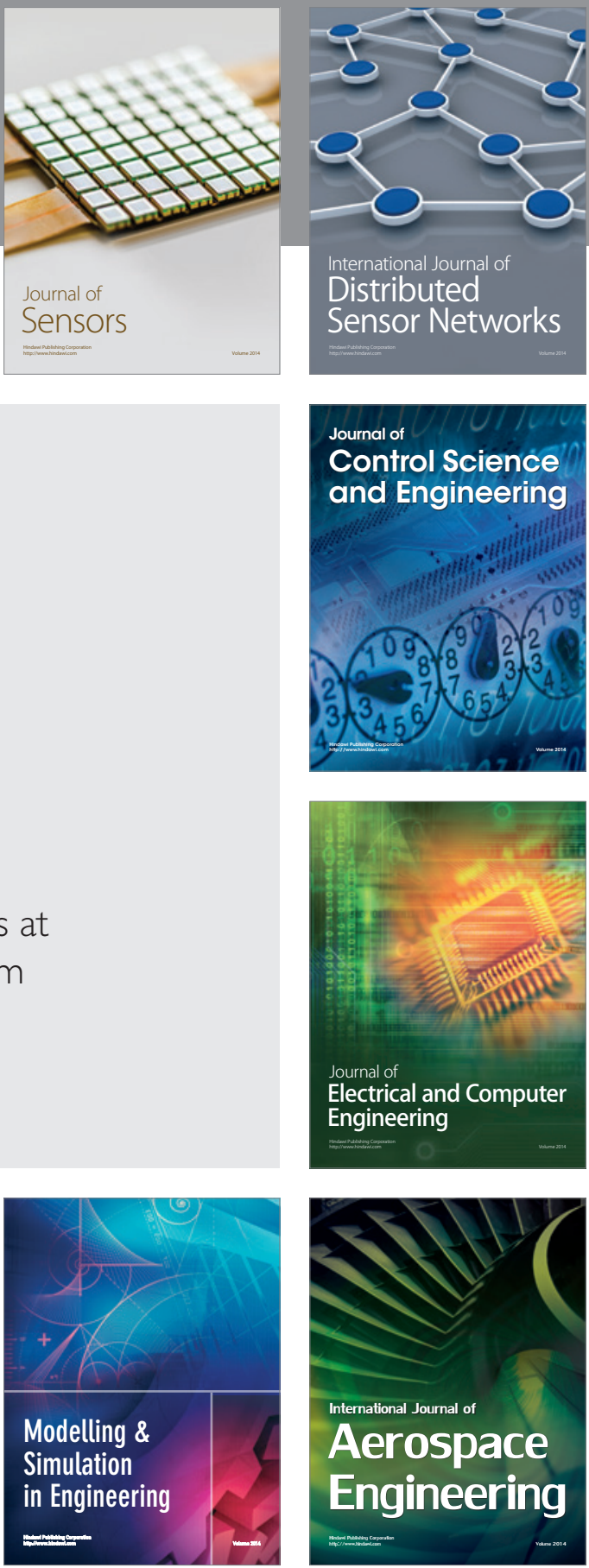

Journal of

Control Science

and Engineering
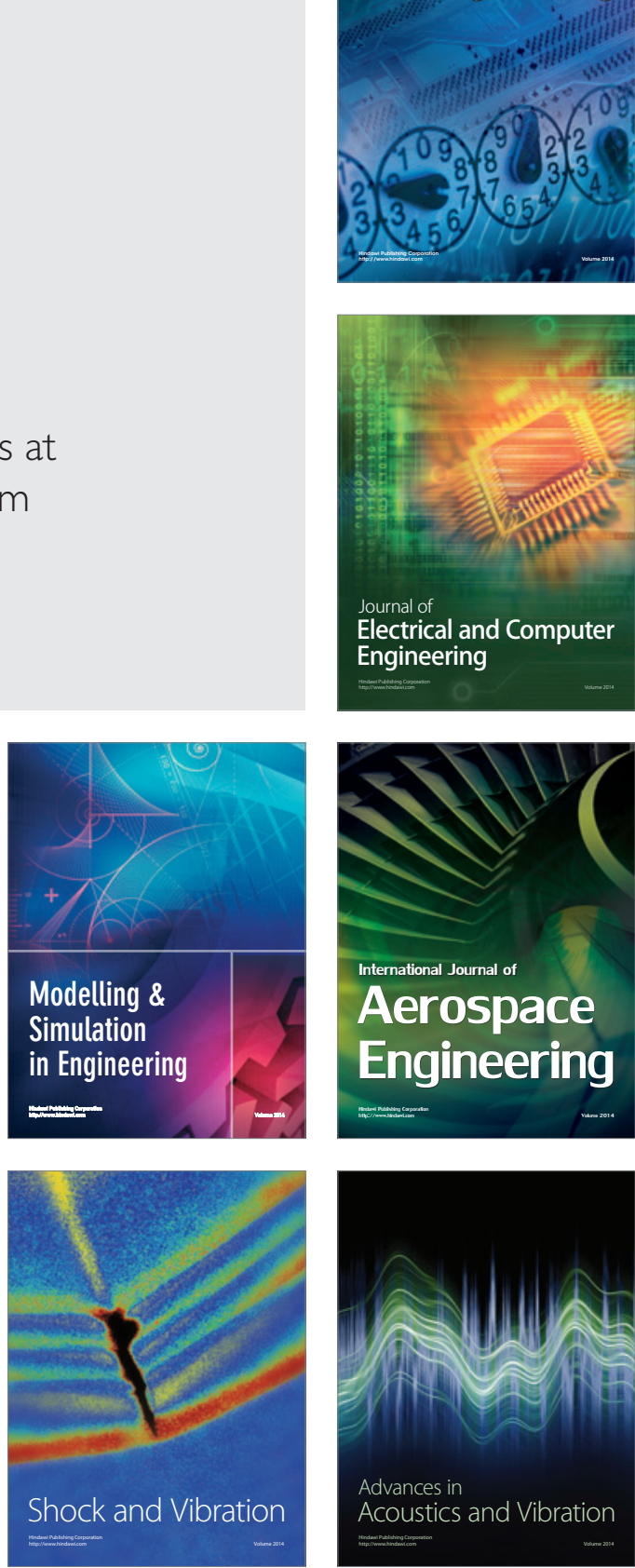